\title{
Advanced Abdominal Pregnancy Following in Vitro Fertilization and Embryo Transfer: A Case Report
}

\section{Osato Giwa-Osagie ${ }^{1}$, Benedict Ekaidem ${ }^{1,}$, , Bobo Kayode ${ }^{2}$, Bolanle Are ${ }^{1}$, Philomena Aisagbohni ${ }^{2}$, Charles Oniha ${ }^{3}$}

${ }^{1}$ Department of Obstetrics \& Gynecology, Omni Medical Centre and Advanced Fertility Clinic, Lagos, Nigeria

${ }^{2}$ Advanced Fertility Unit, Omni Medical Centre and Advanced Fertility Clinic, Lagos, Nigeria

${ }^{3}$ Department of Paediatrics, Omni Medical Centre and Advanced Fertility Clinic, Lagos, Nigeria

\section{Email address:}

drbenekaidem@yahoo.com (B. Ekaidem)

\section{To cite this article:}

Osato Giwa-Osagie, Benedict Ekaidem, Bobo Kayode, Bolanle Are, Philomena Aisagbohni, Charles Oniha. Advanced Abdominal Pregnancy Following in Vitro Fertilization and Embryo Transfer: A Case Report. Journal of Gynecology and Obstetrics. Vol. 4, No. 1, 2016, pp. 1-6. doi: 10.11648/j.jgo.20160401.11

\begin{abstract}
Background: An abdominal pregnancy is a rare condition with high morbidity and mortality. Unlike most other ectopic pregnancies, it has a unique potential to progress to fetal viability. Reports abound of live births following spontaneously-conceived abdominal pregnancies but same cannot be said of those conceived via In vitro fertilization/Embryo transfer (IVF/ET). We report a case of an undiagnosed abdominal pregnancy following IVF/ET, which resulted in a live birth. Case presentation: A 50-year-old booked Gravida 2 Para $0+1$ lady with an IVF pregnancy was admitted at 28 weeks gestation based on complaints of vague abdominal pains. All Ultrasound scans performed prior to and after admission reported the pregnancy as intrauterine. While on admission, she developed preeclampsia which became severe necessitating an urgent abdominal delivery at 34 weeks. The 'urgent caesarean section' eventually became a laparotomy. A live female fetus weighing $2.4 \mathrm{~kg}$ was extracted from the peritoneal cavity. The placenta which was implanted mainly on the omentum was resected and removed. Both the mother and her baby were discharged home in good conditions. Conclusion: An Abdominal pregnancy may occur following IVF/ET and be missed during antenatal care despite repeated ultrasound examinations. Clinicians must maintain a high index of suspicion for its occurrence.
\end{abstract}

Keywords: Ectopic Pregnancy, Assisted Conception, Ultrasound Scan, Abdominal Pain, Laparotomy, Placenta, Omentum, Live Fetus, Ivf/Et

\section{Introduction}

Abdominal pregnancy is a form of ectopic pregnancy defined by the occurrence of an intra-peritoneal implantation exclusive of tubal or ovarian implantation [1]. Although rare, abdominal pregnancy is usually complicated and leads to high maternal morbidity and mortality as well as fetal wastage.

Generally, about 1 percent of all pregnancies are extrauterine pregnancies [1] out of which about 1-3 percent are abdominal pregnancies [1,2]. The reported global incidence of abdominal pregnancies ranges from 1 per 10,000 to 1 per 20,000 pregnancies [3-6]. In Nigeria however; an incidence of 1 per 654 deliveries has been reported [7]. The maternal mortality rate of abdominal pregnancy has been reported to be 7.7 times higher than that of tubal pregnancy and 90 times higher than that of intrauterine pregnancy while the perinatal mortality rate is between $40 \%$ and $95 \%$ [8].

Theoretically, an abdominal pregnancy is believed to occur due to intra-abdominal fertilization of a spermatozoon and an ovum or as a result secondary implantation from an aborted tubal pregnancy [9]. Instead of the typically non-conducive extra uterine environment preventing further development as seen with other ectopic pregnancies, an abdominal pregnancy occasionally defies the odds and may advance to fetal viability with a favorable outcome [2].

In spite of the advancement in diagnostic tools, it is still common to find reports of abdominal pregnancy being diagnosed at an advanced stage of pregnancy and sometimes incidentally at delivery $[5,7,10,11]$. Therefore, clinicians should maintain a high index of suspicion in 
patients with suggestive risk factors, signs or symptoms.

Since In vitro fertilization is a known risk factor for ectopic pregnancies, the current increased recourse to it for infertility treatment may mean that the frequency of ectopic pregnancies may also be on the rise. It is therefore important that practitioners entertain the possibility of complications such as abdominal pregnancy following IVF/ET.

We hereby report a case of an advance abdominal pregnancy following in vitro fertilization resulting in a favorable feto-maternal outcome, in order to draw the attention of IVF practitioners and healthcare givers as a whole to its possibility.

\section{Case Report}

A 50 year old Gravida 2 para $0+1$ lady was admitted at a gestational age 29 weeks on account of complaints of vague abdominal pains. About 3 months prior to this, she had been experiencing milder episodes of similar pain but observed that it had become slightly worse few hours to presentation. There was no history of vaginal bleeding, no urinary or bowel symptoms. She perceived satisfactory fetal movements.

Her conception was via in vitro fertilization and embryo transfer using donor oocytes and her husband's spermatozoa. Two embryos at blastocyst stage were transferred on 10/09/2014. An ultrasound scan performed on $12 / 11 / 2014$ had revealed a single viable intrauterine gestation with crown rump length of $52.5 \mathrm{~mm}$ which was equivalent to a gestational age of 12 weeks. At 14 weeks of gestation she had an elective cervical cerclage (using McDonald's technique), and thereafter was on routine antenatal care until the $29^{\text {th }}$ week when she was admitted.

There was no prior history of diagnosis or treatment for any chronic medical illness. In 2013, she also had conceived via IVF/ET but had the pregnancy medically terminated at 19 weeks following an anomaly scan diagnosis of fetal meningocoele.

Examination at presentation revealed a patient who was in some painful distress and mildly pale. She was afebrile not dehydrated and not icteric. Her pulse rate was 80 beats per minute, blood pressure measured $110 / 70 \mathrm{mmHg}$ and her chest was clinically clear. The abdomen was gravidly enlarged and not tender. The fetus was in oblique lie and cephalic presentation. The fetal heart beat was present and rate was $140 \mathrm{bpm}$. There was no palpable uterine contraction.

The preliminary investigations conducted were: obstetric ultrasound scan, cardiotocograph, and complete blood count. Other investigations requested were: blood film for malaria, urinalysis and urine culture. The significant findings were anemia (hemoglobin estimation of $8 \mathrm{~g} / \mathrm{dl}$ ), presence of malaria infestation and finding on ultrasound scan of major type placenta previa. Based on these, she was admitted into the ward for conservative management. She was transfused with 3 pints of whole blood and treated for malaria with Arthemeter/Lumefantrine (Coartem ${ }^{\circledR}, \quad$ Novartis). Her abdominal pain was reduced by oral paracetamol, although she was still in some mild abdominal discomfort. It was decided that she should be on bed rest and close monitoring until her delivery based on the aforementioned features. All obstetric ultrasound scans performed failed to show the reason for her discomfort.

Approximately a week following admission, she developed preeclampsia which later became severe. Antihypertensive drugs were commenced including Alpha methyl dopa, Nifedipine and Labetalol. Her blood pressure ranged between $140 / 80$ and $170 / 100 \mathrm{mmHg}$. Urinalysis revealed significant proteinuria $(2+$ on dipstick). Close fetal surveillance was ensured with Ultrasound scans and Cardiotocography. Intramuscular Corticosteroid (2 doses of Dexamethasone 24hours apart) was administered in order to enhance fetal lung maturity. A decision was made to deliver her by cesarean section at 34 weeks based on the diagnosis of severe preeclampsia, major type placenta previa and the patient's symptomatology. Four units of whole blood were crossmatched in anticipation of possible hemorrhage from placenta previa. Also, an informed consent was obtained for the procedure.

Intra-operatively, a Pfannenstiel incision was made and further deepened into the peritoneal cavity with the patient under subarachnoid block. The following findings were noted: (1) A thickened peritoneum, (2) Sudden gush of greenish fluid (meconium-stained liquor) on entering the peritoneal cavity, (3) A live female fetus lying transversely in the peritoneal cavity. She weighed $2.4 \mathrm{~kg}$ at birth and had an APGAR score of 8 and 9 in the first and fifth minutes respectively, (4) Placenta mainly attached to the greater omentum with a small portion attached to the serosa of the uterine fundus, (5) A bulky uterus in the pelvis with a fenestration of approximately $4 \mathrm{~cm}$ on the fundus, (6) Grossly normal tubes and ovaries, (7) Generalized filmy adhesions all over the peritoneal cavity.

On entering the peritoneal cavity, the fetal lower limbs were grasped and the infant delivered breech. She was immediately handed over the pediatrician in attendance for resuscitation. The umbilical cord stump was traced down to the placental bulk and the placental attachment sites noted. It was noted that that the placenta was mostly attached to the omentum with a little tip to the uterine fundus. The other attachments to the peritoneum were flimsy and easily separable. The omentum was mobilized, partially resected and removed together with the placental bulk. The filmy adhesions of the placenta to the peritoneum and other structures were bluntly separated. Adequate hemostasis was ensured. The total blood loss was estimated as 800 millilitres. The peritoneal cavity was lavaged with warm saline and a peritoneal drain left in place. The abdominal wound was closed routinely in layers after which the cervical cerclage was removed through the vagina. The patient was subsequently transfused with 4 pints of whole blood.

Post operatively, the patient's clinical condition remained stable. Examination on first post-operative day revealed a satisfactory general condition. Her pulse rate ranged between 
79 to $89 \mathrm{bpm}$ while her blood pressure range was $140 / 90$ to $120 / 80 \mathrm{mmHg}$. Her $\mathrm{SPO}_{2}$ was $98 \%$. Urinary output was satisfactory. The abdomen was soft and not unduly tender. The peritoneal drain was only productive of 40 millilitres of sero-sanguineous fluid and bowel sounds were present. By second post-operative day, peritoneal drain was removed, and oral intake was commenced. Her haemoglobin estimation on the second post-operative day was $9 \mathrm{~g} / \mathrm{dl}$. Subsequently, her condition remained satisfactory and she was discharged home on the $8^{\text {th }}$ post-operative day.

The neonatologist examined the infant thoroughly and found that the child had talipes equinovarus of the left foot. She also developed intermittent apneic attacks which necessitated admission. While on admission, the child received blood transfusion and was placed on supplemental oxygen. She was eventually weaned of oxygen and discharged home after a week of hospital admission. The child was referred to orthopedic surgeons for further review and management of the talipes equinovarus. Apart from the talipes equinovarus, there were no other congenital anomalies.

\section{Discussion}

The case described highlights 3 important facts namely: the possibility of abdominal pregnancy occurring following IVF/ET, the challenge with early diagnosis and thirdly, the possibility of a live birth from an abdominal pregnancy.

There have been previous reports of abdominal pregnancy occurring after IVF/ET [12-14]. However, none of these reported cases resulted in a favorable fetal outcome. The case presented therefore does not only buttress the fact that this condition can follow a carefully controlled process like IVF/ET; it further shows that a favorable outcome is attainable with abdominal pregnancies derived from IVF/ET.

The continuing difficulty in diagnosing this potentially life threatening condition is clearly highlighted in this case. The fact that it occurred after an early ultrasound had confirmed the pregnancy to be intrauterine probably led to the lack of suspicion even when there were few suggestive signs and symptoms. The suggestive clinical features identified at hind sight include persistent abdominal pain with no identifiable cause, painful fetal movements, abnormal fetal presentations and unexplained anemia. Several other ultrasound scans were performed by different doctors both within and outside the managing hospital that also missed the diagnosis. Currently, Magnetic Resonance Imaging (MRI) and Computerized Tomography Scan (CTScan) have been advocated for since about $50 \%$ of the diagnoses are missed on ultrasound [15]. Another useful aid is maternal serum alpha fetoprotein assay value which may be abnormally elevated without explanation in women with abdominal pregnancies [16]. These other tests were not done probably because the possibility of the condition was not entertained clinically

Management of advanced abdominal pregnancy appears to be slightly controversial. There is a dilemma as to whether immediate surgical termination should be offered in order to reduce maternal mortality or an expectant approach adopted with the aim of achieving successful fetal outcome. Some clinicians argue that if there is an ongoing abdominal pregnancy greater than 24 weeks, a conservative approach should be taken to allow fetal maturity and improve chances of survival [17]. While it is unlikely that the delivery of this patient could have been delayed had the diagnosis been known, the successful outcome achieved in this case, together with many others reported with advance abdominal pregnancies calls for a rethink. Based on the experience of this case, one tends to agree that expectant management may be a feasible option if the pregnancy is closely monitored. The patient's condition should however be optimized and the clinician ready for emergency intervention at all times.

In anticipation of torrential bleeding at surgery, crossmatched blood should be made available as well as other measures to tackle massive hemorrhage. The patient presented was fortunate to have been so prepared because of the presence of other risk factors for hemorrhage even when the diagnosis was missed. These risks were severe pre eclampsia, placenta previa and preexisting anemia.

Morbidity and mortality from abdominal pregnancy is generally believed to be closely related to the management of the placenta. Intra-operatively, a decision has to be made to either remove or leave the placenta in situ. Fortunately, surgical management of this patient was made easy because the bulk of the placenta was attached to the omentum whose blood vessels were easily mobilized and ligated without damage to other visceral organs. If this is not feasible the placenta should be left in situ with the umbilical cord ligated and then followed up expectantly [18]. For the latter category of patients, the post-operative period is usually turbulent as complications such as infection, abscesses, bowel obstruction secondary to adhesions or wound dehiscence is said to occur in about half of the patient [19]. In addition, the use of methotrexate or selective arterial embolization is said to help in placental resorption [19].

An abdominal pregnancy is often associated with fetal deformities [20] such as facial and cranial asymmetry, joint abnormalities and limb deformity, and central nervous deformities in about $21 \%$ of cases. In our case, the pediatrician reported the finding of a lower limb deformity (talipes equinovarus).

\section{Conclusion}

The importance of this case report is the fact that an abdominal pregnancy can occur following in vitro fertilization /embryo transfer, and could be missed during antenatal care despite repeated ultrasound examinations. Emphasis should be placed on thorough clinical assessment, evaluation of patients and maintenance of a high index of suspicion for its occurrence. Furthermore, this case lends credence to the possibility of adopting an expectant approach in the management of carefully selected cases of advance abdominal pregnancies, as a good outcome is achievable. 


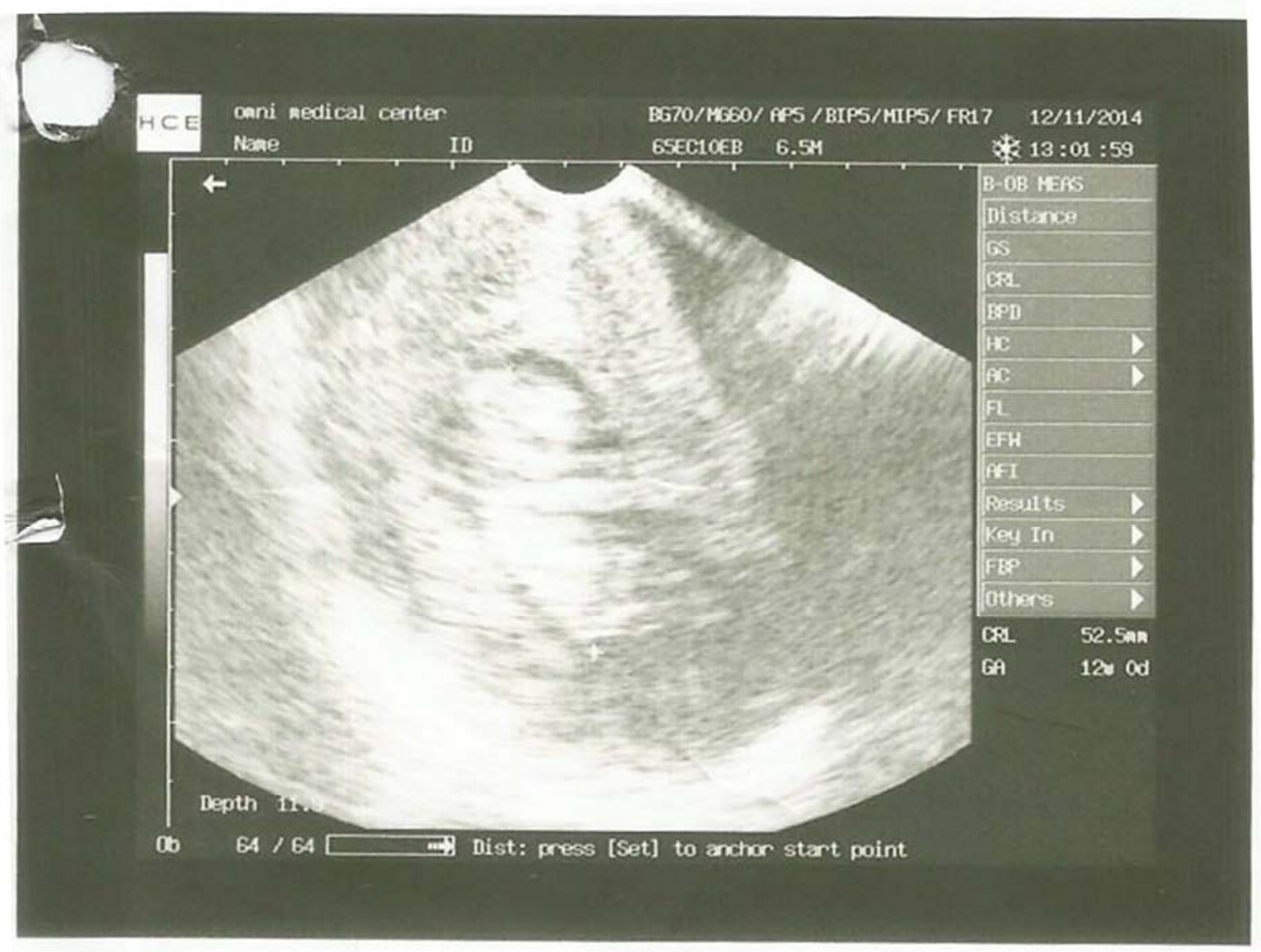

Figure 1. Ultrasound scan picture confirming the pregnancy as intrauterine at 12 weeks of gestation.

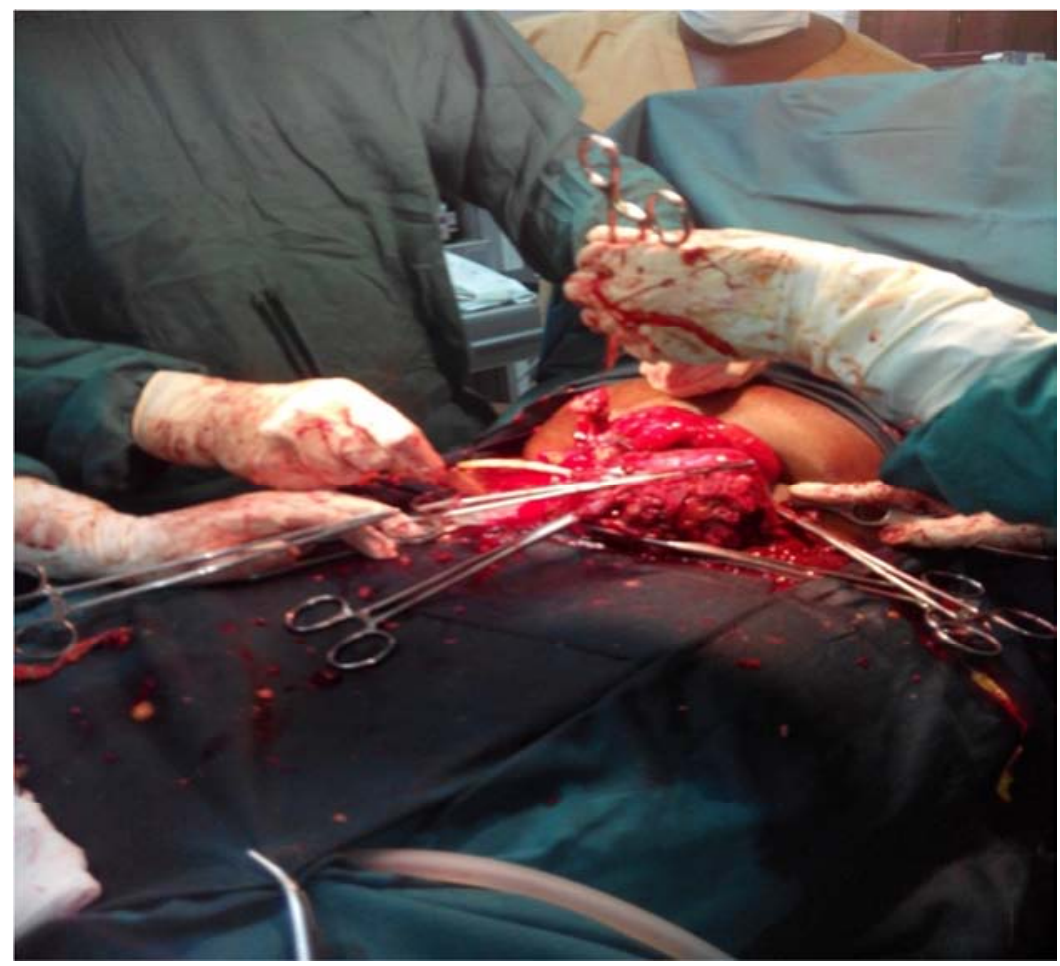

Figure 2. Following the delivery of the fetus, the placenta shown was attached almost entirely to the omentum. The uterus was down in the pelvis (not shown here). 


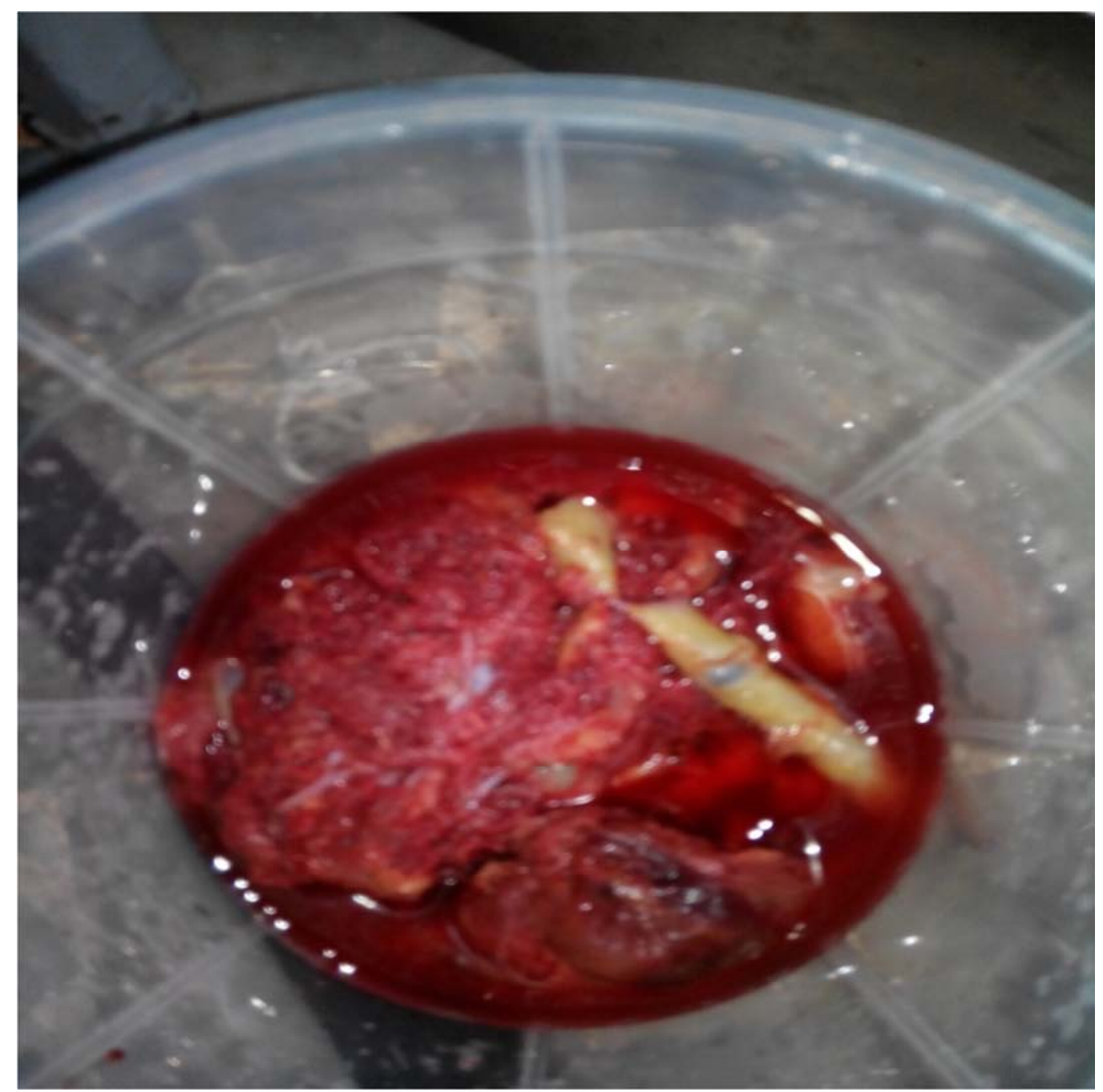

Figure 3. The Placenta.

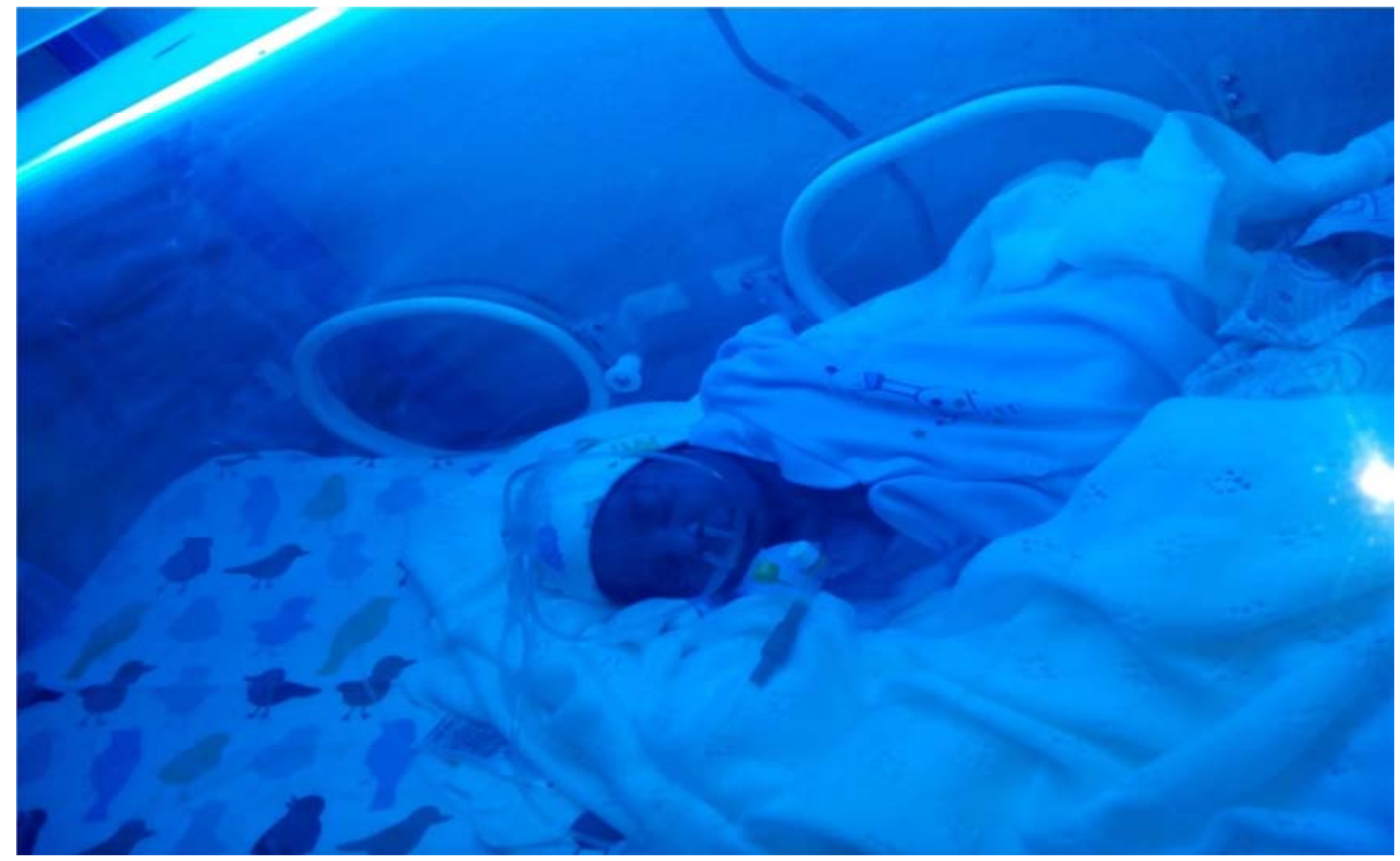

Figure 4. The neonate immediately after resuscitation. 


\section{References}

[1] Molinaro TA, Barnhart KT. Semin Reprod Med. 2007; 25(2): 123-130.

[2] Parekh VK, Bhatt S, Dogra VS. JUM. 2008; 27: 4679-681.

[3] Ayinde OA, Aimakhu CO, Adeyanju OA, Omigbodun AO. Abdominal pregnancy at the University College Hospital, Ibadan: a ten-year review. Afr J Reprod Health. 2005; 9(1): 123-7.

[4] Atrash HK, Friede A, Hogue CJR. Abdominal pregnancy in the United States: frequency and maternal mortality. Obstet Gynecol 1987; 69: 633-7.

[5] Bang Ntamack JA1, Ngou Mve Ngou JP, Sima Ole B, Sima Zue A, Mayi Tsonga S, Meye JF. Abdominal pregnancy in Libreville from 1999 to 2009. J Gynecol Obstet Biol Reprod (Paris). 2012 Feb; 41(1): 83-7.

[6] Nwobodo EI. Abdominal pregnancy. A case report. Ann Afr Med. 2004; 3(4): 195-6.

[7] Tasnim N1, Mahmud G. Advanced abdominal pregnancy--a diagnostic and management dilemma. J Coll Physicians Surg Pak. 2005; 15(8): 493-5.

[8] Badria L, Amarin Z, JaradatA, ZahawiH,Gharaibeh A. Full term viable abdominal pregnancy, a case report and review. Arch Gynaecol Obstet. 2003; 268(4): 340-2.

[9] Varma R, Mascarenhas R, Jame D. Successful outcome of advanced abdominal pregnancy with exclusive omental insertion. Ultrasound Obstet Gynecol 2003; 21: 192-194.

[10] Baffoe P, Fofie C,Gandau BN. Term abdominal pregnancy with a healthy newborn. Ghana Med J. 2011; 45(2): 81-3.
[11] Dover R W. Powell M C. Management of a primary abdominal pregnancy. Am J Obstet Gynecol 1995; 172: 16031604.

[12] Moonen-Delarue MW, Haest JW. Ectopic pregnancy three times in line of which two advanced abdominal pregnancies. Eur J Obstet Gynecol Reprod Biol. 1996; 66(1): 87-8.

[13] Fisch B, Peled Y, Kaplan B, Zehavi S, Neri A. Abdominal pregnancy following in vitro fertilization in a patient with previous bilateral salpingectomy. Obstet Gynecol. 1996; 88(4 Pt 2): 642-3.

[14] Cormio G, Santamato S, Putignano G, Bettocchi S, Pascazio F. Concomitant abdominal and intrauterine pregnancy after in vitro fertilization in a woman with bilateral salpingectomy. A case report. J Reprod Med. 2003; 48(9): 747-9.

[15] Shafi SM, Malla MA, Salaam PA, Kirmani OS. Abdominal pregnancy as a cause of hemoperitoneum. J Emerg Trauma Shock. 2009; 2(3): 196-8.

[16] Bombard AT, Nakagawa S, Runowicz CD, Cohen BL, Mikhail MS, Nitowsky HM. Early detection of abdominal pregnancy by maternal serum AFP + screening. Prenat Diag 1994; 14: 1155-7.

[17] Hage ML, Wall LL, Killam A. Expectant management of abdominal pregnancy. A report of two cases. J Reprod Med 1988; 33: 407-10.

[18] Mekki Y, Gilles JM, Mendez L, O'Sullivan MJ. Abdominal pregnancy: To remove or not to remove the placenta. Prim Care Update Ob Gyns 1998; 5: 192.

[19] Martin JN Jr, McCaul JF 4th. Emergent management of abdominal pregnancy. Clin Obstet Gynecol 1990; 33: 438-47.

[20] Stevens A. Malformations and deformations in abdominal pregnancy Am J Med Genet C Semin Med Genet. 1993; 47 (8): 1189-95. 